\title{
Research on Parameters' Determination and Imaging of CT System
}

\author{
Nan Qiao ${ }^{1}$, Jiu Xiong ${ }^{2, *}$ and Qi Huang ${ }^{3}$ \\ School of Mathematical Sciences, Ocean University of China, Qingdao, Shandong, 266100, China \\ ${ }^{*}$ Corresponding author
}

\begin{abstract}
In this paper, to determine parameters of the CT system and reconstruct images, the geometric model of the template is used to calculate its parameters, and the image is reconstructed based on the Filtered back projection algorithm, which contains the RADON inverse transform and the usage of calibrated CT system parameters. To remove the noise of the image background, we optimize the image of CT with discrete properties of pixels. What is more, this paper presents a new template that is more appropriate to determine CT system parameters. And it is more conducive to calculating the unit spacing and the receiving angle of detectors, and therefore this new system improves the calibration accuracy of CT system parameters.
\end{abstract}

Keywords-filtered back projection algorithm; radon inverse transformation; tomography; CT system parameters; permutation function

\section{INTRODUCTION}

CT (Computed Tomography), which is the computerized tomography scan, can use the energy absorption characteristics of samples to perform tomographic imaging of biological tissue and engineering material samples without destroying the sample, thereby obtaining internal structural information of the sample. As a computerized tomography technology, it plays an increasingly important role in many fields such as medicine, industry, geophysics, agriculture and engineering detection. However, there are inevitable errors in the installation of CT systems, which affects the imaging quality. Therefore, it is necessary to calibrate the parameters of installed CT system, and it means the parameters of the CT system are calibrated by means of the sample of known structure (called a template). And according to this, the samples of the unknown structure are imaging.

In this paper, taking the data from the Mathematical Contest in Modeling (2017), for the calibration of CT system parameters and the reconstruction of images, the parameters of the template are determined based on the geometric properties of the template, and the image is reconstructed based on the filtered back projection algorithm, and the imaging is optimized based on the discrete nature of the pixels. At the same time, this paper presents a template that can easily calibrate CT system parameters.

\section{RESEARCH IDEAS}

\section{A. Calibration of the CT system Parameters}

First, we should analyze the geometric information of the template (as shown in the figure below), which contains unit spacing, angle, and center of rotation. There is a small round in the template, and its radius is much smaller than the minor axis of the given ellipse, so the projection of the small circle could be clearly identified in most of the receiving information of the template. And according to the principle that the projection length of the circle in any direction is unchanged, we could approximately calculate the spacing by the known radius of the small circle and the number of detectors which could detect these small circles. In addition, we could also clearly distinguish the projection of the ellipse from most of the received information in the template. Therefore, the receiving angle and center of rotation could be derived. In this way, the parameters of the CT system could be calibrated: detector spacing, rotation angle, and rotation center.

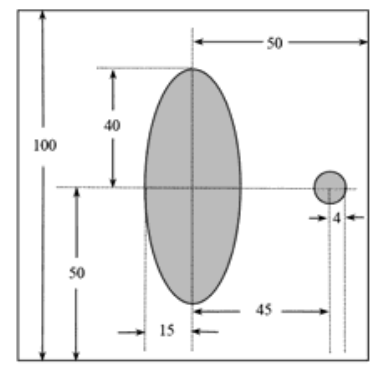

FIGURE I. TEMPLATE SCHEMATIC DIAGRAM

\section{B. Image Reconstruction}

We choose to use the filtered back projection algorithmRADON inverse transform and calibrated CT system parameters for image reconstruction. If the unknown medium is evenly distributed, the image is ideal, on the other hand, if the unknown medium is not evenly distributed, there are impurities in the background of the reconstructed image, which reduces the visibility of the image.

At this point, the permutation function is defined to quickly filter out the noise in the images' background. As a result, it has been found that after optimizing the image with this permutation function, the image sharpness is greatly improved

\section{Template Design}

For these many disadvantages of parameter determination of this CT system in this question, we designed a template that is easy to calibrate parameters. Three points were fulfilled when designing a new template: 1 . Simple enough 2 . Easy to calculate the cell spacing of detectors 3 . It could accept the angle and its change sensitively. 


\section{MODEL ESTABLISHMENT AND SOLUTION}

\section{A. Geometric Model of the Template}

To simplify the model, let the length of minor axis be $l$, and the length of major axis be $a$. It is known that the length of the projection of the ellipse onto the line $l$ is $p$, and the slope of the straight line $k$ is obtained (if $k$ does not exist, the receiving angle is $90^{\circ}$ ). If $k$ is obtained, the receiving angle $\alpha$ can be calculated, and then the rotation center $C\left(x_{0}, y_{0}\right)$ is obtained by the projection distance $D$ from the center of the detector to the center of the ellipse and the multiple groups of $\alpha$.

- Solution of unit spacing $d$ of detector

From the given data, we could get that the number of detectors which could detect these small circles is 28 or 29 , and

$$
\mathrm{d} \approx 0.2759 \mathrm{~mm}
$$

- The solution of the geometric model of the template The slope of the line:

$$
k=\sqrt{\frac{d^{2}-4 a^{2}}{4-d^{2}}}
$$

- Receiving angle

$$
\alpha=\arctan k=\arctan \sqrt{\frac{d^{2}-4 a^{2}}{4-d^{2}}}
$$

- Rotation times and receiving angles:

\begin{tabular}{cccccc}
\hline time & angle & time & angle & time & angle \\
\hline 1 & 29.968 & 60 & 92.296 & 120 & 148.451 \\
10 & 38.895 & 70 & 97.621 & 130 & 158.824 \\
20 & 48.609 & 80 & 108.769 & 140 & 168.227 \\
30 & 58.995 & 90 & 118.640 & 150 & 179.297 \\
40 & 68.532 & 100 & 128.596 & 160 & 189.120 \\
50 & 78.266 & 110 & 138.470 & 170 & 199.073 \\
\hline
\end{tabular}

Suppose that the detector is regarded as the radiation from the first detector to the second detector, the angle between the ray and the positive half of the $x$ axis is defined as the acceptance angle, and all receiving angles could be obtained. Then the center of rotation Take the center of the tray as the origin, so we could get the rotation center:

$$
C(-9.1147,6.0787)
$$

The blank part of the following figure is because the data with the large error is manually removed.

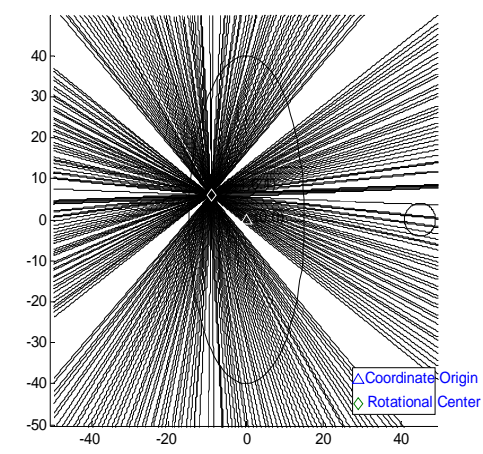

FIGURE II. TRAJECTORY OF THE VERTICAL LINE IN THE DETECTOR

\section{B. The Radon Inverse Transformation Model}

- Radon inverse transformation

$$
f(x)=\left(\frac{1}{2 \pi^{2}}\right) \int_{0}^{\pi} \int_{-\infty}^{+\infty} \frac{\left[\frac{\partial g}{\partial s}(s, \theta)\right]}{x \cos \theta+y \sin \theta-s} d s
$$

The above formula reveals the basic method of image reconstruction in $\mathrm{CT}$ technology. The matrix of receiving information $A_{k}$ is known, and according to the geometric model of the template, we could solve the receiving angle $\alpha_{j}$ of $A_{k}(i j)$ :

$$
p\left(i, \alpha_{j}\right)=A_{k}(i j)
$$

$p(s, \alpha)$ is got from $I_{k}(x, y)$ (which is the absorption function) by the Radon reverse transformation, so we could use the interpolation and inverse transformation to calculate the absorption function and the absorption matrix. [1]

Explanation: Absorption function refers to absorption rate of unknown medium in the two-dimensional plane

\section{Image Reconstruction}

Directly using the filtered back projection algorithm (Radon inverse transform), we could get the image as follows: [2]

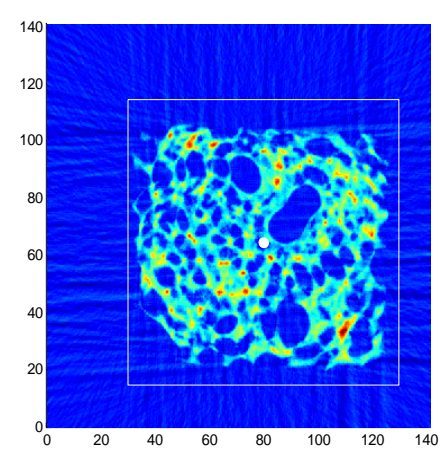

FIGURE III. THE ABSORPTION IMAGE (UNTREATED)

Since this unknown medium to is a non-uniform object, there is a lot of noise in the background, and the visibility of the image is low. Then we define the permutation function to quickly eliminate the noise of this picture. After obtaining the discrete matrix of the absorptivity of the unknown medium, it is 
rearranged and then graphed. And the permutation function $f(x)$ is defined:

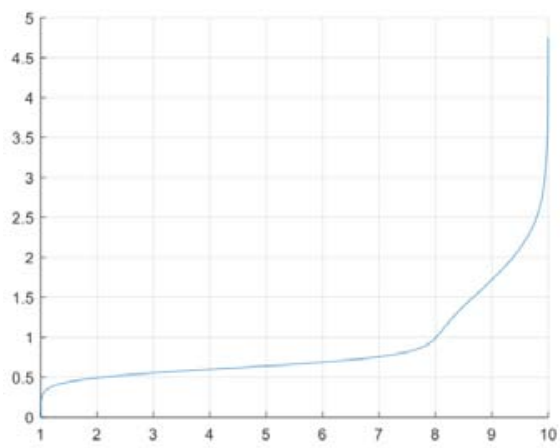

FIGURE IV. THE PERMUTATION FUNCTION

$f(x)$ is a discrete function that arranges all numerical points of the absorption matrix from small to large in the domain interval $[0,10]$. In general, the absorption rate of the background noise is lower than that of the real medium. And the absorption rate of these points which belong to the background noise could be concentrated in one interval. [5] What is more, the value of the interval function $f$ is usually much smaller in the other interval, thus the maximum absorptivity of background noise can be determined

To evaluate the effect of inverse transformation:

$$
\left\{\begin{array}{l}
w=\int_{\text {Noise interval }} f(x) d x \\
r=\int_{\text {domain of definition }} f(x) d x
\end{array}\right.
$$

Then

$$
g_{1}=\frac{w}{r}
$$

$g_{1}$ is the discriminant score. It reflects the proportion of the filtrated noise. If the value is too small, it may indicate that the filtering effect is not good, on the other hand, it may indicate that some important details are lost. When defining the absorptivity, adjusting the minimum absorptivity above the background noise will not cause much interference.

After using the template to receive the information and find the absorbance of the template, calculate the permutation function. And then we reuse the Radon inverse transform and permutation function to get the reconstructed image as follows, and we could find that the final imaging results are good.:

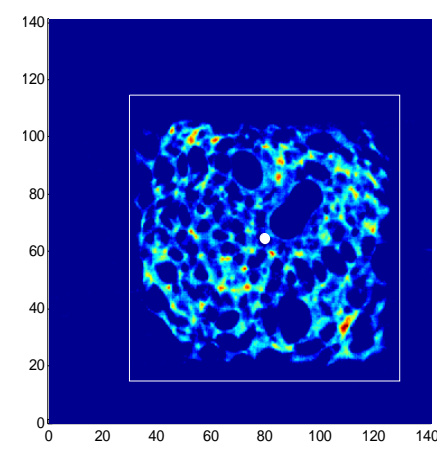

FIGURE V. OPTIMIZED IMAGE

\section{Improvement of Calibration Parameters' Selection}

In fact, the absorption matrix of the background noise of each unknown medium is different from that of the template absorption matrix. To automatically determine the background noise, all these numbers in absorption matrix are converted into the positive numbers and we should maintain the ratio of each value and map the range of $f(x)$ to $[0,1]$, and then analyze its differential value. Because of the special nature of each medium, it is still necessary to manually determine the background noise. [3] Because the number of unknown media is small, all background noise should be determined by human for accuracy.

Using the background noise of the template is not sufficient to correct the medium with inhomogeneous distribution, and it is necessary to manually select new background noise.

\section{ERROR ANALYSIS}

The main source of model error is in the process of inferring the projection length from the received information. Whether it is to calculate the cell spacing of the detectors or to calculate the length of the elliptical projection, using the detector as the length measurement tool is the main source of all errors.

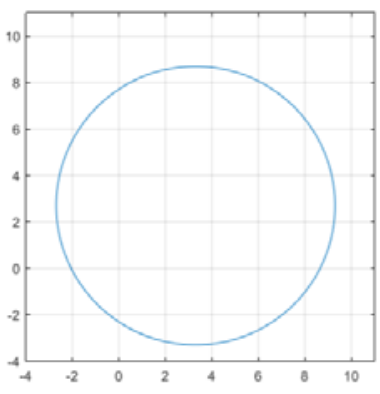

FIGURE VI. SAMPLE

If the grid lines in this figure are regarded as X-rays, and the two axes serve as detectors (one detector per every unit). The number of detectors that observe the projection is 6 , and then the projection length obtained by this measurement is 5 , while the actual diameter of this circle is 6 . Because there are many times of observations, the detector unit spacing could be accurately derived. The unit spacing of the detector is one unit. The diameter of the small circle is 27 or 28 in the received information. The diameter of the small circle is less than 29 and greater than 28 , and then we choose 29 , because it is easy to calculate. 
The maximum relative error of the unit spacing $(d)$ of the detector is

$$
S_{d}=\frac{\frac{8}{28}-\frac{8}{29}}{\frac{8}{28}} \times 100 \% \approx 3.4483 \%
$$

The result is within the allowable range of errors. In conclusion, a better image is simulated by the obtained rotation center, receiving angle and distance between detector units, and it could be considered that the error has no significant effect on the model.

\section{MODEL IMPROVEMENT}

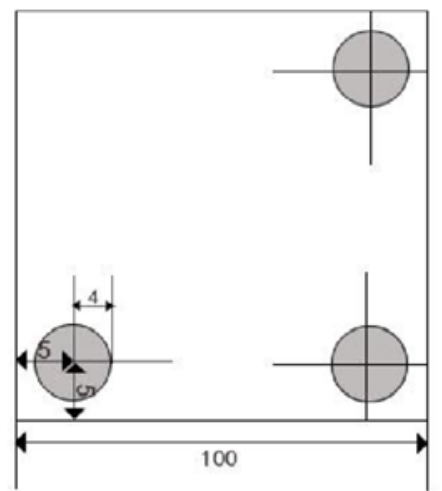
FIGURE VII. THE GEOMETRIC DIAGRAM OF THE NEW
TEMPLATE

The picture above is a newly-designed two-dimensional template image in which there are uniform templates and a blank background. For measuring the unit spacing of the detector, the shape of all the medium with absorptivity in the image is circular. Meanwhile, for the convenience of measuring the receiving angle of the detector, there is no circle at the topleft corner.

Take the center of the square as origin, horizontally right direction as $x$-axis positive direction and vertically upward direction as $y$-axis positive direction.

Define the coordinate of the center of rotation as $(-10,10)$, the receiving angle as $30^{\circ}$ to $209^{\circ}$, and detect the data every other $1^{\circ}$.

Confirmed by Radon transform, the result is shown in the Fig. 8 below. By observing the position where three circles overlap, the message that the newly-simulative template have received can confirm that the detector is directly to the right of the template when it rotates for about 60 th time. For about 105 th time, it is perpendicular to the line between two circles, one at the bottom-left corner and another at the top-right. For about 150th time, it is right over the template. Therefor it could be confirmed that the detector rotates anticlockwise. Before they intersect for the second time, use the distance between the two circles at bottom-left and bottom-right corner to compute the angle, and after they intersect for the second time, use the distance between the two circles at bottom-right and top-right corner to compute the angle. But the result is unsatisfactory, differing by about $5^{\circ}$ from the real angle. It may be because computing arc cosine when the two circles are close to each other will enlarge the error.

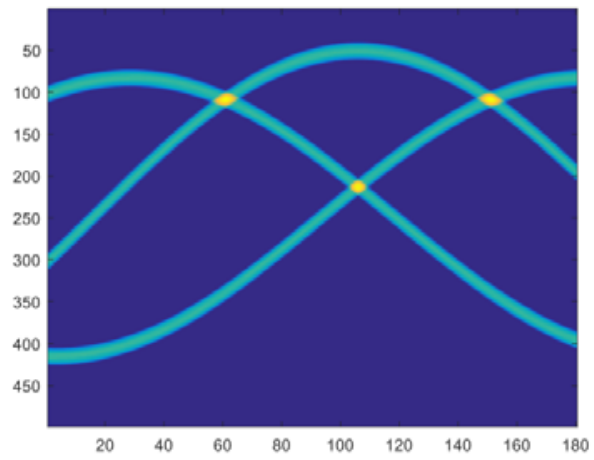

FIGURE VIII. SIMULATED NEW TEMPLATE RECEIVES INFORMATION

\section{CONCLUSION}

Radon transform and its inverse transform are widely used in exploration activities and 3D object recognition. The Radon inverse transform evaluation model in this paper could be used to quickly and effectively resolve background noise interference and improve the recognition accuracy.

\section{REFERENCES}

[1] A three-dimensional-weighted cone beam filtered backprojection (CBFBP) algorithm for image reconstruction in volumetric CT - Helical scanning. Tang, Xiangyang,Hsieh, Jiang,Nilsen, Roy A.,Dutta, Sandeep,Samsonov, Dmitry,Hagiwara, Akira. Physics in Medicine and Biology . 2006

[2] Superimposition of 3D cone-beam CT models of orthognathic surgery patients. Cevidanes L H S,Bailey L J,Tucker G R,Styner M A,Mol A,Phillips C L,Proffit W R,Turvey T. Dento maxillo facial radiology . 2005

[3] A three-dimensional weighted cone beam filtered backprojection (CBFBP) algorithm for image reconstruction in volumetric CT under a circular source trajectory. Tang, Xiangyang,Hsieh, Jiang,Hagiwara, Akira,Nilsen, Roy A.,Thibault, Jean-Baptiste,Drapkin, Evgeny. Physics in Medicine and Biology . 2005

[4] Enhanced 3-D-reconstruction algorithm for C-arm systems suitable for interventional procedures. Wiesenl, Karl,Barth, K.,Navab, N.,Durlak, P.,Brunner, T.,Schuetz, O.,Scissler, W. IEEE Transactions on Medical Imaging . 2000

[5] SHORT-SCAN FAN-BEAM ALGORITHMS FOR CT. Naparstek,Abraham. IEEE Transactions on Nuclear Science . 1980

[6] OPTIMAL SHORT SCAN CONVOLUTION RECONSTRUCTION FOR FANBEAM CT. D I Parker. Journal of Computational Finance 1982

[7] Image reconstruction from fan-beam projections on less than a short scan. Noo, Frédéric,Defrise, Michel,Clackdoyle, Rolf,Kudo, Hiroyuki. Physics in Medicine and Biology . 2002

[8] Feldkamp-type VOI reconstruction from super-short-scan cone-beam data. Yu Hengyong, Wang Ge. Medical Physics . 2004

[9] Exact helical reconstruction using native cone-beam geometries. Noo, Frédéric,Heuscher, Dominic. Physics in Medicine and Biology . 2003

[10] Practical cone-beam algorithm. Feldkamp LA,Davis LC,Kress JW. Journal of the Optical Society of America A: Optics, Image Science and Vision

[11] Practical cone-beam algorithm. Feldkamp LA,Davis LC,Kress JW. Journal of the Optical Society of America A: Optics, Image Science 